\title{
Full-Spectrum Photonic Pigments with Non- iridescent Structural Colors through Colloidal Assembly
}

\section{Citation}

Park, Jin-Gyu, Shin-Hyun Kim, Sofia Magkiriadou, Tae Min Choi, Young-Seok Kim, and Vinothan N. Manoharan. 2014. "Full-Spectrum Photonic Pigments with Non-Iridescent Structural Colors through Colloidal Assembly." Angew. Chem. Int. Ed. 53 (11) (February 12): 2899-2903. doi:10.1002/anie.201309306.

\section{Published Version}

doi:10.1002/anie.201309306

\section{Permanent link}

http://nrs.harvard.edu/urn-3:HUL.InstRepos:24873725

\section{Terms of Use}

This article was downloaded from Harvard University's DASH repository, and is made available under the terms and conditions applicable to Open Access Policy Articles, as set forth at http:// nrs.harvard.edu/urn-3:HUL.InstRepos:dash.current.terms-of-use\#OAP

\section{Share Your Story}

The Harvard community has made this article openly available.

Please share how this access benefits you. Submit a story.

Accessibility 


\title{
Full-Spectrum Photonic Pigments with Non-iridescent Structural Colors through Colloidal Assembly
}

\author{
Jin-Gyu Park, Shin-Hyun Kim, Sofia Magkiriadou, Tae Min Choi, Young-Seok Kim, Vinothan N. \\ Manoharan*
}

Coloration free from chemical- or photo-bleaching is a central goal in paints, cosmetics, and information displays. Such long-lasting colors can be achieved by making non-absorbing nanostructures that selectively reflect light of certain wavelengths through constructive interference of scattered waves. This phenomenon is called structural coloration [1]. Colloidal suspensions are ideal for making structurally colored materials, since they are inexpensive, they can be manipulated dynamically with external fields [2], and they have optical properties that can be tuned through synthesis, morphology, and the suspension medium [3]. The primary motivation for the work presented here is the production of photonic pigments that can be used for particle-based reflective displays with high efficiency. Grayscale, field-addressable "electronic-ink"-type displays have been produced from microcapsule pigments containing black colloidal particles that absorb light and white particles that scatter light [4]. Full-color reflective displays require colorful microcapsule pigments that reflect light efficiently and isotropically, so that the color does not vary with the viewing angle in the device. In principle such colors can be produced from amorphous packings of colloidal particles [5], which do not suffer from the angledependence, or iridescence, of crystalline packings of colloids, the subject of many previously reported photonic pigment schemes [6]. However, processes for synthesizing large quantities of photonic

\footnotetext{
Dr. J.-G. Park, S. Magkiriadou, Prof. V. N. Manoharan*

School of Engineering and Applied Sciences

Department of Physics

Harvard University

17 Oxford St. MA 02138 (USA)

E-mail: vnm@seas.harvard.edu

Homepage: http://manoharan.seas.harvard.edu

Prof. S.- H. Kim, T. M. Choi

Department of Chemical and Biomolecular Engineering,

Korea Advanced Institute of Science and Technology Daejeon, 305-701 (Korea)

\author{
Dr. Y.-S. Kim \\ Korea Electronic Technology Institute, \\ 68 Yatap-dong, Bundang-gu, Seongnam-si \\ Gyeonggi-do (Korea)
}

This research is supported by an International Collaboration grant (No. Sunjin-2010-002) from the Ministry of Trade, Industry \& Energy of Korea. We also acknowledge support from the Harvard MRSEC through NSF grant no. DMR0820484. This work is performed in part at the Center for Nanoscale Systems (CNS) at Harvard University, which is supported by the National Science Foundation under NSF award no. ECS-0335765. We also thank Prof. Gi-Ra Yi from Sungkyunkwan University for helpful discussion.
}

pigments based on amorphous arrays of colloidal particles are only beginning to emerge $[7,8]$.

The principal challenge of making isotropic structural coloration through colloidal assembly is control over incoherent and multiple scattering. Previous approaches produce bulk coatings of colloidal particles a few micrometers thick, corresponding roughly to the extinction length of the nanostructures [7]. If the sample is too thick, white color from multiple scattering dominates, compromising the color saturation. Furthermore, as the size of the colloidal particles increases, the structural resonance is compromised by incoherent scattering at shorter wavelengths [8]. Therefore, it remains a challenge to produce full-spectrum (blue, green, red) isotropic photonic pigments. Another technical challenge lies in making the amorphous nanostructures. Charged nanoparticles such as polystyrene or silica tend to crystallize as the concentration increases [9]. Alternatives are to use bidisperse suspensions or to rapidly concentrate the suspensions to avoid crystallization, but both routes complicate the processing $[7,8]$.

Here we present a new colloidal assembly method to fabricate micro-encapsulated photonic pigments that can cover the full spectrum with little multiple scattering. The key to our approach is to design a pigment with three different structural length scales: the scatterer size, the inter-scatterer distance, and the capsule size. We use microfluidic techniques to produce spherical microcapsules approximately $100 \mu \mathrm{m}$ in diameter that contain densely packed, amorphous arrays of colloidal particles. The encapsulated particles are polystyrene/poly( $N$-isopropylacrylamide-co-acrylic-acid) (PS /poly-NiPAm-AAc) core-shell particles in which the shells are closely index-matched to the surrounding fluid (water). As shown in our previous work [10], the use of core-shell particles allows us to independently tune the scattering of the particles, which is set by the core size, and the wavelength of the structural resonance, which is determined by the inter-scatterer distance and controlled by the shell diameter. We show that capsules containing amorphous suspensions of core-shell particles have weak incoherent and multiple scattering. Therefore the coherent scattering responsible for the structural color dominates at all inter-scatterer distances, and we are able to make photonic pigments that show isotropic structural colors throughout the visible range.

We make these photonic pigments using a microfluidic processing technique that yields uniform microcapsules with amorphous internal structures. We start with an aqueous suspension of PS/poly-NiPAm-AAc core-shell particles synthesized through seeded emulsion polymerization [11] using PS nanoparticles of hydrodynamic radius $R_{h P S}=78 \pm 5 \mathrm{~nm}$ as seeds. The ratio of NiPAm monomer to seed particle, which controls the thickness of the soft polyNiPAm shell, is $0.5 \% \mathrm{w} / \mathrm{w}$, yielding a core-shell hydrodynamic radius $R_{h \text { core-shell }}=168 \pm 17 \mathrm{~nm}$. We match the refractive index of the shell to that of the suspension medium by copolymerizing $4.0 \% \mathrm{w} / \mathrm{w}$ of acrylic acid (AAc) in the polyNiPAm shell. We then encapsulate this suspension ( $\phi_{\text {core-shell }} \sim 0.28$ ) in the innermost phase of 
(a)

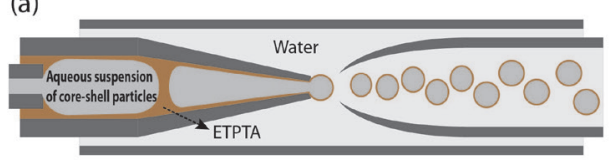

(b)

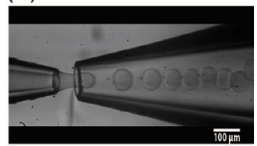

(c)

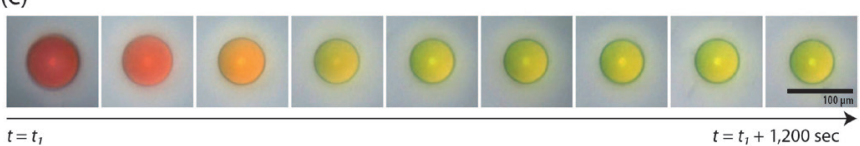

(d)
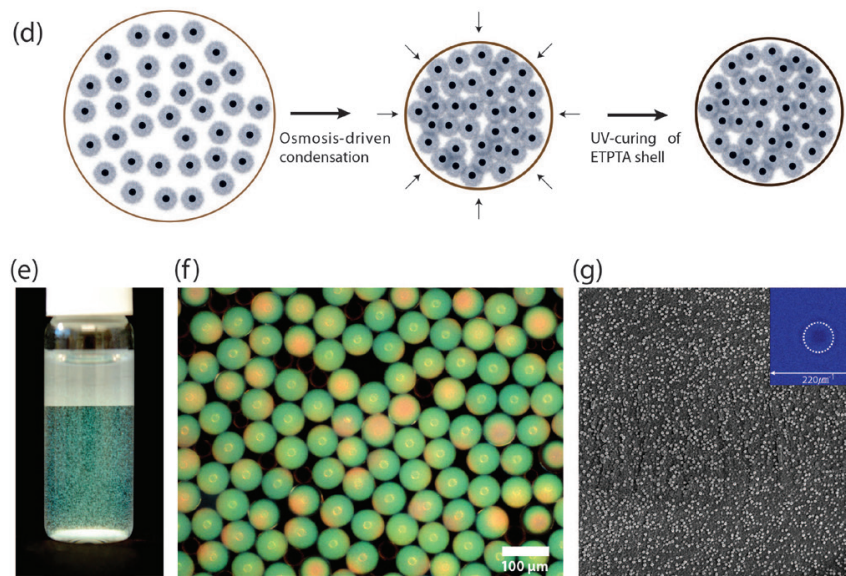

(f)

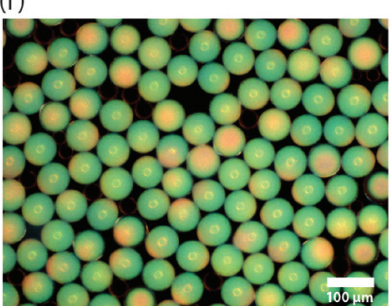

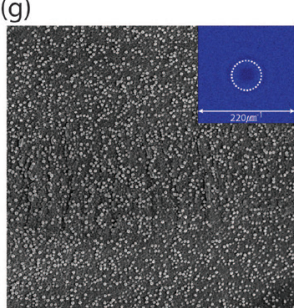

Figure 1. Fabrication of photonic pigments. (a) Schematic of a capillary microfluidic device for the production of W/O/W doubleemulsion droplets with a thin ethoxylated trimethylolpropane triacrylate (ETPTA) membrane. (b) Optical micrograph showing generation of uniform W/O/W droplets with the innermost phase containing an aqueous suspension of core-shell particles with volume fraction $\phi_{\text {core-shell }} \sim 0.28$. (c) Time-series optical micrographs of osmosis-driven condensation of droplets at $340 \mathrm{mOsm} / \mathrm{L}$, where $t_{1}$, the elapsed time for microscope analysis, is $\sim 5 \mathrm{~min}$. (d) Schematic of the structure of particles during osmosis-driven condensation. (e), ( $f$ ) A photograph and an optical micrograph of the photonic pigments in water after UV-curing of the ETPTA shell. The sample is compressed at $440 \mathrm{mOsm} / \mathrm{L}$. (g) Scanning electron micrograph of a cross-section of a cryogenically fractured photonic pigment microcapsule prepared under compression at $440 \mathrm{mOsm} / \mathrm{L}$. The field of view is $20.0 \mu \mathrm{m}$ wide. The inset in $(\mathrm{g})$ is two-dimensional Fourier power spectrum derived from $(g)$.

water-in-oil-in-water $(\mathrm{W} / \mathrm{O} / \mathrm{W})$ double-emulsion droplets using a capillary microfluidic device, as shown schematically in Figure 1(a) [12]. The aqueous suspension and an oil phase containing a UVcurable monomer, ethoxylated trimethylol-propane triacrylate (ETPTA), are injected simultaneously through a hydrophobic capillary, creating a train of suspension droplets in the continuous monomer flow. The hydrophobic nature of the capillary prevents the droplets from wetting. This core-sheath is then emulsified into the continuous phase, aqueous poly(vinyl alcohol) (PVA, 10\%, w/w), at the tip of the capillary, yielding $\mathrm{W} / \mathrm{O} / \mathrm{W}$ double-emulsion drops where the innermost phase is the core-shell particles suspension and the middle phase is an ultra-thin shell of oil, as shown in Figure 1(b) These are the precursors to our photonic microcapsules.

These precursor droplets are specifically designed to enable the facile preparation of amorphous nanostructures. The double emulsion makes it possible to concentrate the suspension by an osmotic pressure gradient, as shown in previous work [13]. Furthermore, the soft shells of the particles help to inhibit crystallization when the suspension is concentrated [14]. We concentrate the particles by placing the $\mathrm{W} / \mathrm{O} / \mathrm{W}$ droplets into an aqueous solution of PVA and sodium chloride $(\mathrm{NaCl})$, creating a positive osmotic pressure difference that forces the water out through the thin ETPTA membrane and causes the droplets to shrink isotropically, as shown in Figure 1(c). As the droplets shrink, the particle concentration increases, and the droplets develop color that blue-shifts as the condensation proceeds (Figure 1(c)). We then polymerize the ETPTA middle layer by exposing it to ultraviolet (UV) light for 30 seconds. This process is illustrated in Figure 1(d). The ETPTA layer $\left(n_{\text {ETPTA }}=1.4689\right)$ yields a $0.8-\mu \mathrm{m}$-thick, optically transparent shell after curing. The resulting spherical photonic microcapsules are highly uniform in size and display noniridescent structural colors, as shown in Figures 1(e) and (f).

The internal structure of these photonic pigments is random and isotropic, as shown by scanning electron microscope images of cryogenically fractured microcapsules (Figure S1(a)). Twodimensional Fourier analysis of a $20 \mu \mathrm{m} \times 20 \mu \mathrm{m}$ cross-section of the microcapsule (Figure 1(g)) shows a ring pattern (inset in Figure $1(\mathrm{~g}))$, indicating that the structure is isotropic with no preferred direction. We observe a characteristic peak corresponding to a length scale of $0.217 \mu \mathrm{m}$, as denoted by the dotted circle in the inset.

We can control the structural colors by changing the average distance between the PS cores, which are the primary scatterers. The inter-scatterer distance is controlled by the degree of droplet compression, which depends on the osmotic pressure difference used to concentrate the particles. For example, we can prepare red photonic microcapsules at $180 \mathrm{mOsm} / \mathrm{L}$, yellow at $260 \mathrm{mOsm} / \mathrm{L}$, and green at $440 \mathrm{mOsm} / \mathrm{L}$. At each osmolarity, emulsion droplets containing core-shell particles with $R_{h \text { core-shell }}=168 \pm 17 \mathrm{~nm}$ are incubated for two hours to equilibrate the osmotic pressure before we polymerize the ETPTA shell. Reflectance spectra of the resulting photonic microcapsules (Figure 2(a)) show that the characteristic peak shifts from $610 \mathrm{~nm}$ to $550 \mathrm{~nm}$ as the osmotic pressure increases, but the normalized full width at half-maximum (FWHM), $\Delta \lambda / \lambda_{\max }$, remains almost constant (Figure 2(b)).

We estimate the inter-scatterer distance $d$ from the reflection spectra. If the particles inside the microcapsules are packed amorphously, and if we assume back-reflected light,

$$
d=0.6 \lambda / n_{\text {medium }}
$$

where $\lambda$ is the resonant wavelength and $n_{\text {medium }}$ is the effective refractive index of the medium [15], which depends on the volume fraction of the PS cores [10]. Equation (1) assumes that the average spacing $d$ between particles in a colloidal glass is determined by the location of the first peak of the structure factor of colloidal glasses at similar particle densities [16]. Because we do not know a priori the volume fraction of polystyrene, we calculate $d$ using an iterative process: we first use Eq. (1) to calculate $d_{0}$ with $n_{\text {medium }}$ equal to the refractive index of water, then use $d_{0}$ to estimate the volume fraction of polystyrene, $\phi_{P S}=\left(d_{\text {core }} / d_{0}\right)^{3}$ and $n_{\text {medium }}$, and from Eq. (1) calculate a new $d_{1}$ from $n_{\text {medium }}$. The values for $d$ converge quickly, and we find $n_{\text {medium }} \approx 1.39$, a physically reasonable value. From this calculation, we determine the number density of PS scatterers and the average distance between them as a function of osmotic pressure (Figure 2(c)). We find that, for the green photonic microcapsules, the inter-scatterer distance calculated from the spectrum $(236 \mathrm{~nm})$ agrees well with that obtained through Fourier analysis of an SEM image of the same sample $(217 \pm 14 \mathrm{~nm})$ On the basis of the spectra, calculations, and SEM analysis, we conclude that the particles are compressed into roughly $35 \%$ of their original volume at $440 \mathrm{mOsm} / \mathrm{L}$ and are arranged in an amorphous structure. 
(a)

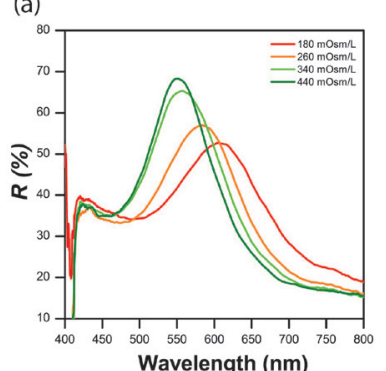

(b)

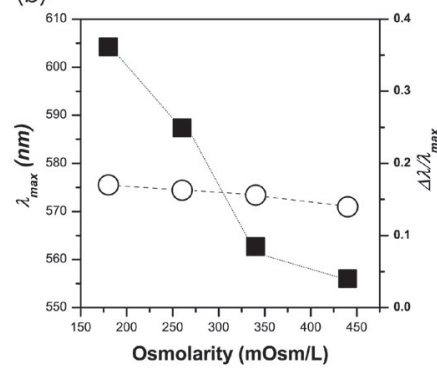

(d)

(c)
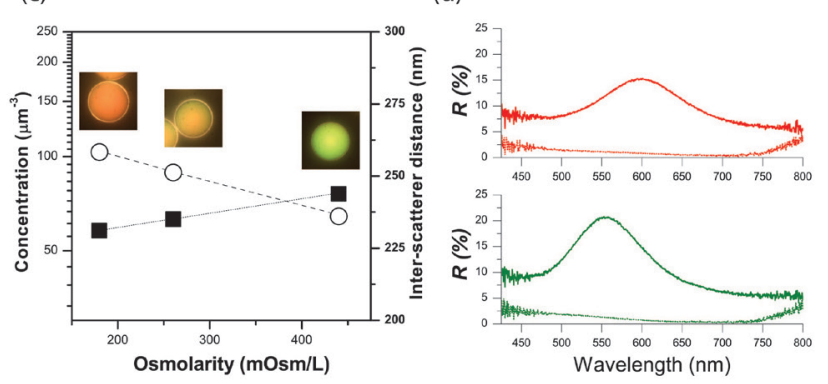

scattering and that the contribution of multiple scattering to the signal is the same in both the co- and cross-polarized spectra. We find values of 15.6 for green and 16.7 for red microcapsules, (a)

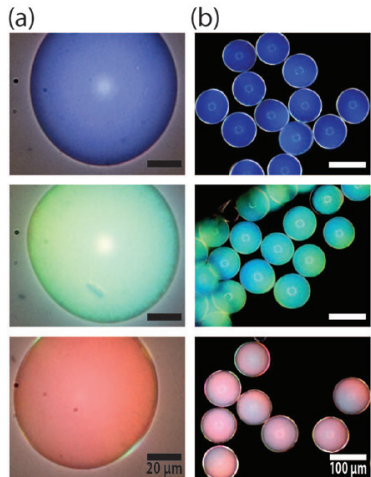

(c)

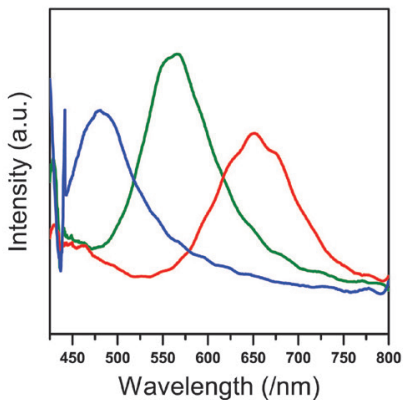

Figure 3. Photonic microcapsules with blue, green, and red structural colors prepared with different shell thickness of core-shell particles. (a) Bright-field and (b) dark-field optical micrographs of photonic microcapsules. (c) Reflectance spectra of the resulting photonic microcapsules. In this synthesis, all the photonic droplets are incubated at $440 \mathrm{mOsm} / \mathrm{L}$ for $1 \mathrm{hr}$ before ETPTA polymerization.

Figure 2. Control of structural colors through osmotic pressure. (a) Reflectance spectra of the photonic microcapsules equilibrated under different osmotic pressures. (b) Plots of peak positions (dark squares) and normalized full-width at half maximum (FWHM, open circles) of the photonic microcapsules as a function of osmotic pressure. (c) Estimated concentration of PS scatteres (dark square) and average spacing between PS scatterers (open circle) as a function of osmotic pressure. Insets are bright-field optical micrographs of red, yellow, and green photonic microcapsules prepared under $180 \mathrm{mOsm} / \mathrm{L}$, $260 \mathrm{mOsm} / \mathrm{L}$, and $440 \mathrm{mOsm} / \mathrm{L}$, respectively. The field of view for the insets is $100 \mu \mathrm{m}$ wide. (d) Co-polarization (lines) and crosspolarization (dots) reflection spectra of red (upper) and green (lower) photonic microcapsules.

In all of these samples, the coherent scattering on resonance dominates the background scattering off resonance. We characterize the background by the intensity at short wavelengths (420-435 nm), where the reflectance has the highest value outside the resonance peak. This background arises from incoherent scattering from individual particles and multiply scattered light. The ratio of the peak intensity to background, $I_{\text {max }} / I_{\text {background, }}$, increases from 1.35 for red photonic microcapsules to 1.83 for green (see Figure 2(a)). This quantity is one measure of the color saturation: the lower the value, the less pronounced the structural color and the whiter the sample, due to mixing of the resonant color with incoherently scattered blue light. Although the off-resonance incoherent scattering is not negligible, particularly for red microcapsules, it is nonetheless significantly smaller than the resonant contribution for all of these samples.

We isolate the contribution of multiply scattered light to the incoherent scattering by measuring the polarization dependence of the reflection spectra, since multiply scattered light loses memory of its original polarization. We linearly polarize the incident white light and place another linear polarizer in front of the detector (Figure S2). When the two polarizers are parallel (co-polarization) we observe peaks in the spectra at approximately $560 \mathrm{~nm}$ for green microcapsules and $610 \mathrm{~nm}$ for red microcapsules (Figure 2(d)), whereas the cross-polarized spectra for both samples are nearly flat across the visible region. From these measurements we calculate the ratio of singly to multiply scattered light at the resonant wavelength, assuming that the entire cross-polarized signal is due to multiple indicating that multiple scattering does not contribute significantly to the structural color. We also calculate the contribution of multiple scattering to the background from the average reflectivity through crossed $\left(I_{c r}\right)$ and parallel $\left(I_{c o}\right)$ polarizers at short wavelengths (420$435 \mathrm{~nm}), I_{\text {cross }} /\left(I_{\text {co }}+I_{\text {cross }}\right)$. We find that multiple scattering accounts for only $22 \%$ of the background intensity in green microcapsules and $25 \%$ in red microcapsules, indicating that the primary source of background scattering is incoherent single scattering from the particles themselves. From these measurements we conclude that multiple scattering does not compromise the color saturation in our system, in contrast to other systems with isotropic structural colors where multiple scattering has to be suppressed through absorption $[7,8]$.

To complete the full spectrum of colors, we make microcapsules from core-shell particles of different sizes. We use the same osmotic compression and the same core sizes for all samples, so that the inter-scatterer distance is controlled by the thickness of the particle shells. We obtain blue photonic microcapsules with $R_{h_{\text {_core-shell }}}=$ $135 \pm 24 \mathrm{~nm}$, green with $R_{h \text { core-shell }}=168 \pm 17 \mathrm{~nm}$, and red with $R_{h_{-} \text {core-shell }}=210 \pm 15 \mathrm{~nm}$, as shown in Figure 3.

The optical properties of our amorphous microcapsules are qualitatively different from those of similar but crystalline structures, even for samples prepared with comparable particle sizes. For comparison, we prepare crystalline photonic supraballs with a diameter of about $90 \mu \mathrm{m}$ through complete consolidation of $250 \mathrm{~nm}$ PS spheres in W/O droplets [17]. Bright-field optical micrographs in Figure 4(a) and (b) show the dramatic differences between the two samples: the amorphous photonic microcapsules show colors that are uniform across each capsule and from capsule to capsule, whereas the crystalline supraballs show multiple patches of different colors and variations in the patches between supraballs. To quantify these differences, we measure the spectra of single photonic microcapsules and crystalline supraballs as a function of position $(x)$ with a microscope-mounted, fiber-optic spectrometer (Figure 4(c)). The position of the spectral peak remains constant for the photonic microcapsule (Figure 4d), while in the crystalline supraball it shifts by $40 \mathrm{~nm}$ or more (Figure 4e). This behavior arises due to the anisotropy of the crystal, which leads to a variation in the resonant 
(a)
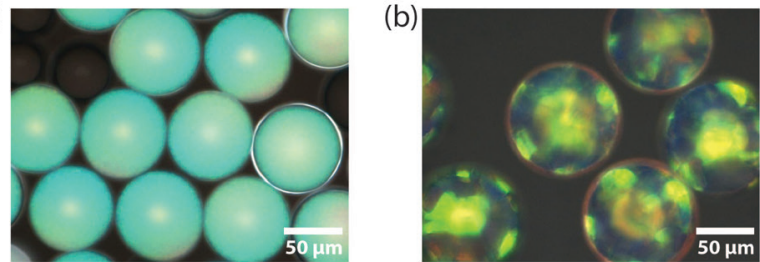

(c)

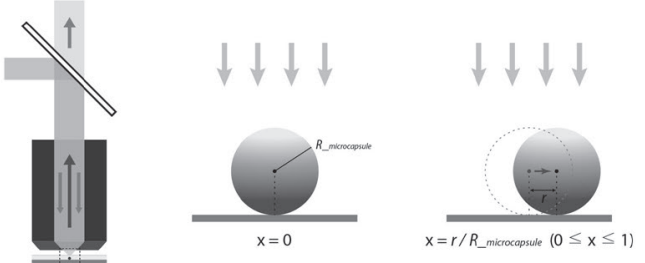

(d)

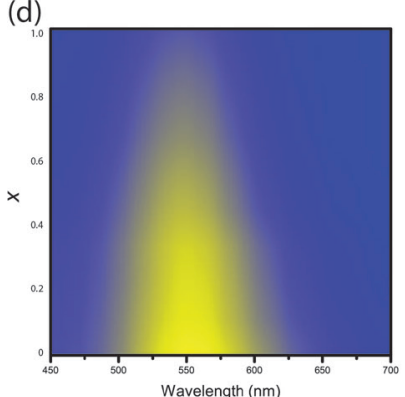

(e)

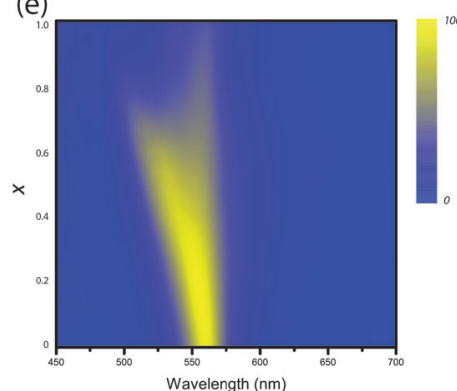

Figure 4. Photonic microcapsules show uniform structural color under different illumination directions. Bright-field optical micrographs of (a) photonic microcapsules and (b) photonic crystalline supraballs. (c) Schematic of the apparatus used to measure the reflection spectra of a single microcapsule or supraball. (d and e) Reflectivity (yellow: $100 \%$, blue: $0 \%$ ) of a single photonic microcapsule (d) and a single photonic crystalline supraball (e) as a function of position $x$, where $x=$ 0 at the center and $x=1$ at the edge.

condition with angle. At the same time, the incompatibility of crystalline order with the spherical symmetry of the droplet likely leads to the formation of grain boundaries, which would account for the variation in colors from supraball to supraball. Our measurements highlight the trade-off in optical performance between crystalline and isotropic structures: while the crystalline supraball has sharper resonant peaks at some locations, the isotropic photonic microcapsule shows a broader but more consistent peak across a wide range of positions. We also measure reflectance spectra of the photonic microcapsules at various angles by rotating the sample stage. As shown in Figure S3 (a), the peak position moves less than $10 \mathrm{~nm}$ and is independent of the viewing angle. Plots of $\Delta \lambda / \lambda_{\max }$ as a function of angle in Figure S3 (b) confirm that the optical properties of our microcapsules are independent of orientation.

In conclusion, we have demonstrated a new colloidal assembly method to fabricate photonic microcapsules with non-iridescent structural colors that cover the entire visible range. The length scales of our system are tuned to suppress multiple scattering, so that coherent scattering is the dominant process determining the color. Future work will focus on increasing the saturation across the visible range; as our experiments show, red microcapsules are not as saturated as green or blue ones due to incoherent single scattering at short wavelengths. Our system provides several potential ways to reduce this incoherent scattering; for example, one could vary the refractive indices of the core and shell, or optimize the ratio of the core size to the inter-particle spacing. The ability to independently tune refractive indices and length scales so as to optimize the color all while maintaining the ability to form amorphous packings make this a promising system for mass-produced photonic pigments.

Keywords: structural color · colloidal assembly $\cdot$ microfluidics isotropic structure $\cdot$ photonic pigment

[1] a) R. O. Prum, R. H. Torres, S. Williamson, J. Dyck, Nature 1998 396, 28-29; b) P. Vukusic, J. R. Sambles, Nature 2003, 424, 852-855; c) S. Kinoshita, S. Yoshioka, ChemPhysChem 2005, 6, 1442-1459; d) M. D. Shawkey, S. L. Balenger, G. E. Hill, L. S. Johnson, A. J. Keyser, L. Siefferman, J. R. Soc. Interface 2006, 3, 527-532; e) V. Saranathan, J. D. Forster, H. Noh, S. -F. Liew, S. G. J. Mochrie, H. Cao, E. R. Dufresne, R. O. Prum, J. R. Soc. Interface 2012, 9, 2563-2580.

[2] a) R. C. Hayward, D. A. Saville, I. A. Aksay, Nature 2000, 404, 5659; b) I. Muševič, M. Škarabot, U. Tkalec, M. Ravnik, S. Žumer, Science 2006, 313, 954-958; c) J. Ge, Y. Hu, Y. Yin, Angew. Chem. Int. Ed. 2007, 46, 7428-7431; d) J. D. Forster, J. -G. Park, M. Mittal, H. Noh, C. F. Schreck, C. S. O’Hern, H. Cao, E. M. Furst, E. R. Dufresne, ACS Nano 2011, 5, 6695-6700.

[3] a) J. F. Bertone, P. Jiang, K. S. Hwang, D. M. Mittleman, V. L. Colvin, Phys. Rev. Lett. 1999, 83, 300-303; b) M. L. Breen, A. D. Dinsmore, R. H. Pink, S. B. Qadri, B. R. Ratna, Langmuir 2001, 17, 903-907; c) C. Graf, D. L. J. Vossen, A. Imhof, A. van Blaaderen, Langmuir 2003, 19, 6693-6700.

[4] a) B. Comiskey, J. D. Albert, H. Yoshizawa, J. Jacobson, Nature 1998, 394, 253-255; b) T. Nisisako, T. Torii, T. Takahashi, Y. Takizawa, Adv. Mater. 2006, 18, 1152-1156.

[5] a) K. Ueno, A. Inaba, Y. Sano, M. Kondoh, M. Watanabe, Chem Commun. 2009, 3603-3605; b) M. Harun-Ur-Rashid, A. B. Imran, T. Seki, M. Ishii, H. Nakamura, Y. Takeoka, ChemPhysChem 2010, 11 , 579-583.

[6] a) S. -H. Kim, S. -J. Jeon, G. -R. Yi, C. -J. Heo, J. H. Choi, S. -M. Yang, Adv. Mater. 2008, 20, 1649-1655; b) S. -H. Kim, S. -J. Jeon, S. -M. Yang, J. Am. Chem. Soc. 2008, 130, 6040-6046; c) S. -H.Kim, S. -J. Jeon, W. C. Jeong, H. S. Park, S. -M. Yang, Adv. Mater. 2008, 20, 4129-4134; d) T. Kanai, D. Lee, H. C. Shum, R. K. Shah, D. A. Weitz, Adv. Mater. 2010, 22, 4998-5002; e) T. Kanai, D. Lee, H.C. Shum, D.A. Weitz, small, 2010, 6, 807-810.

[7] J. D. Forster, H. Noh, S. -F. Liew, V. Saranathan, C. F. Schreck, L. Yang, J. -G. Park, R. O. Prum, S. G. J. Mochrie, C. S. O’Hern, H. Cao, E. R. Dufresne, $A d v$. Mater. 2010, 22, 2939-2944.

[8] Y. Takeoka, S. Yoshioka, A. Takano, S. Arai, K. Nueangnoraj, H. Nishihara, M. Teshima, Y. Ohtsuka, T. Seki, Angew. Chem. Int. Ed. 2013, 52, 7261-7265.

[9] a) P. Jiang, J. F. Bertone, K. S. Hwang, V. L. Colvin, Chem Mater. 1999, 11, 2132-2140; b) O. D. Velev, A. M. Lenhoff, E. W. Kaler, Science 2000, 287, 2240-2243; c) D. J. Norris, E. G. Arlinghaus, L. Meng, R. Heiny, L. E. Scriven, Adv. Mater. 2004, 16, 1393-1399.

[10] S. Magkiriadou, J. -G. Park, Y. -S. Kim, V. N. Manoharan, Opt. Mater. Express 2012, 2, 1343-1352.

[11] A. Perro, G. Meng, J. Fung, V. N. Manoharan, Langmuir 2009, 25, 11295-11298.

[12] S. -H. Kim, J. W. Kim, J. -C. Cho, D. A. Weitz, Lab Chip 2011, 11, 3162-3166

[13] S. -H. Kim, J. -G. Park, T. M. Choi, V. N. Manoharan, D. A. Weitz, in submission

[14] J. Mattsson, H. M. Wyss, A. Fernandez-Nieves, K. Miyazaki, Z. Hu, D. R. Reichman, D. A. Weitz, Nature 2009, 462, 83-86.

[15] H. Noh, S. -F. Liew, V. Saranathan, R. O. Prum, S. G. J. Mochrie, E. R. Dufresne, H. Cao, Opt. Express 2010, 18, 11942-11948.

[16] R. Kurita, E. R. Weeks, Phys. Rev. E 2010, 82, 011403.

[17] S. -H. Kim, S. Y. Lee, G. -R. Yi, D. J. Pine, S. -M. Yang, J. Am. Chem. Soc. 2006, 128, 10897-10904. 


\section{Full-Spectrum Photonic Pigments with Non-iridescent Structural Colors through Colloidal Assembly}

Jin-Gyu Park, Shin-Hyun Kim, Sofia Magkiriadou, Tae Min Choi, Young-Seok Kim, Vinothan N. Manoharan*

Supporting Information including Experimental Section and Figure S1 -3. 


\section{Experimental Section}

Particle synthesis: The core-shell particles are synthesized by a two-step emulsion polymerization process [11]. All materials are used as received. In the first step, $380 \mathrm{mg}$ of sodium lauryl sulfate (SLS, 99\%, Aldrich) and $3.75 \mathrm{~g}$ of $\mathrm{N}$-isopropylacrylamide (NiPAm, 97\%, Aldrich) are dissolved in $262.5 \mathrm{~mL}$ of DI water in a $500 \mathrm{~mL}$ three-necked round-bottom flask equipped with a reflux condenser, a nitrogen inlet and a mechanical stirrer. Then $71.25 \mathrm{~mL}$ of styrene (99\%, Aldrich) are added under vigorous stirring. After heating the mixture to $80^{\circ} \mathrm{C}$, we add $180 \mathrm{mg}$ of potassium persulfate (KPS, 99\%, Aldrich) dissolved in 7.5 $\mathrm{mL}$ of DI water to the reactor. We perform the reaction for 8 hours. We wash the resulting PS nanoparticles by dialysis against DI water for five days. The hydrodynamic radius of the PS core particles is $78 \pm 5 \mathrm{~nm}$, as measured by dynamic light scattering (DLS, ALV SP-125) with a $532 \mathrm{~nm}$ Verdi laser (Coherent).

In the second step, we first mix all ingredients in a $40 \mathrm{~mL}$ glass vial including $478 \mathrm{mg}$ NiPAm; $3 \mathrm{mg}$ N,N'-methylenebis-acrylamide (MBA, molecular biology grade, Promega); $0.019 \mathrm{~mL}$ of acrylic acid (AAc, 99\%, Sigma); 19.0mg of KPS dissolved in $0.7 \mathrm{~mL}$; and $20 \mathrm{~mL}$ of PS core suspension. After mixing, we polymerize by tumbling the vial in a reaction bath for $3 \mathrm{hrs}$ at $80^{\circ} \mathrm{C}$. We control the thickness of the polyNiPAm-AAc shell by varying the concentration of PS cores in the core suspension. For the synthesis of particles with $R_{h_{-} \text {core-shell }}=135 \pm 24 \mathrm{~nm}$, we add $7.5 \%(\mathrm{w} / \mathrm{v})$ of PS core suspension; $5.0 \%$ for $R_{h_{-} \text {core-shell }}$ $=168 \pm 17 \mathrm{~nm}$; and $2.5 \%$ for $R_{h \_ \text {core-shell }}=210 \pm 15 \mathrm{~nm}$.

Preparation of microcapsules: The design of the glass capillary microfluidic device is shown in Figure 1(a). Details of device preparation are described in our previous work $[12,13]$. The orifice of the injection capillary is $140 \mu \mathrm{m}$ in diameter, and that of the collection capillary is $200 \mu \mathrm{m}$ in diameter. These orifices are separated by $150 \mu \mathrm{m}$. To produce double-emulsion droplets, an aqueous suspension of core-shell particles containing 2\% w/w PVA (Mw 13,000 - 23,000, Sigma-Aldrich) is used as the innermost phase, and ETPTA (Sigma-Aldrich) containing $0.2 \% \mathrm{w} / \mathrm{w}$ of photo-initiator (2-hydroxy-2-methyl-1-phenyl-1propanone, Sigma-Aldrich) is used as the middle phase. The continuous phase is a $10 \% \mathrm{w} / \mathrm{w}$ aqueous solution of PVA. Flow rates are typically set to $300 \mu \mathrm{L} / \mathrm{h}$ for the innermost phase, $200 \mu \mathrm{L} / \mathrm{h}$ for the middle, and $3,000 \mu \mathrm{L} / \mathrm{h}$ for the continuous phase, resulting in discontinuous generation of doubleemulsion drops. The double-emulsion drops are incubated in aqueous solutions with three different osmolarities of 180, 260, and $440 \mathrm{mOsm} / \mathrm{L}$ for 2 hours at room temperature, and then exposed to UV light (Innocure $100 \mathrm{~N}$ ) for 30 seconds; the incubation solutions each contain 2\% w/w PVA and 80, 120 or 210 $\mathrm{mM} \mathrm{NaCl}$. The polymerized capsules are then transferred into distilled water. 
Characterization: The internal structure of photonic microcapsule is observed with scanning electron microscopy (SEM). We load suspensions of photonic microcapsules into gold planchets $(3.05 \mathrm{~mm}$, Freezer hats, single cavity) and quickly freeze the sample in liquid nitrogen. The samples are then transferred into the high vacuum coater (Leica MED-020 Baltec Sputter Coater) using a vacuum cryotransfer system $\left(5 \times 10^{-7} \mathrm{mbar}\right.$, Leica EM VCT100). The samples are knife- fractured at $-145^{\circ} \mathrm{C}$ and then warmed to $-90^{\circ} \mathrm{C}$ for $3 \mathrm{~min}$ to sublimate the excess water off and expose the fractured surface of the microcapsule. Once the sample cools back down to $-145^{\circ} \mathrm{C}$, the samples are sputter-coated with a $\mathrm{Pt} / \mathrm{Pd}$ target (80/20, $8 \mathrm{~mm}$ thickness). Then we transfer the coated sample into the scanning electron microscope (Zeiss NVision dual focused ion beam) using the vacuum cryo transfer system. Imaging is performed at an accelerating voltage of $2.0 \mathrm{kV}$. We quantify the colors of photonic microcapsules by measuring their optical reflectance spectra using a fiber optic spectrometer (Ocean Optics Inc., HR2000+) attached to an optical microscope (Nikon, ECLIPSE LV100) via a $600 \mu \mathrm{m}$ broadband optical fiber (Ocean Optics Inc., QP600-2-UV/VIS) positioned at the image plane (Figure S2). We image with a 50x objective (Nikon, LU Plan Fluor, NA $=0.8$ ). We use the reflection signal from a silver-coated mirror (Thorlabs PFSQ10-03$\mathrm{P} 01)$ as a reference, to normalize the spectra of our microcapsules to the spectrum of the halogen white light source of the microscope. 

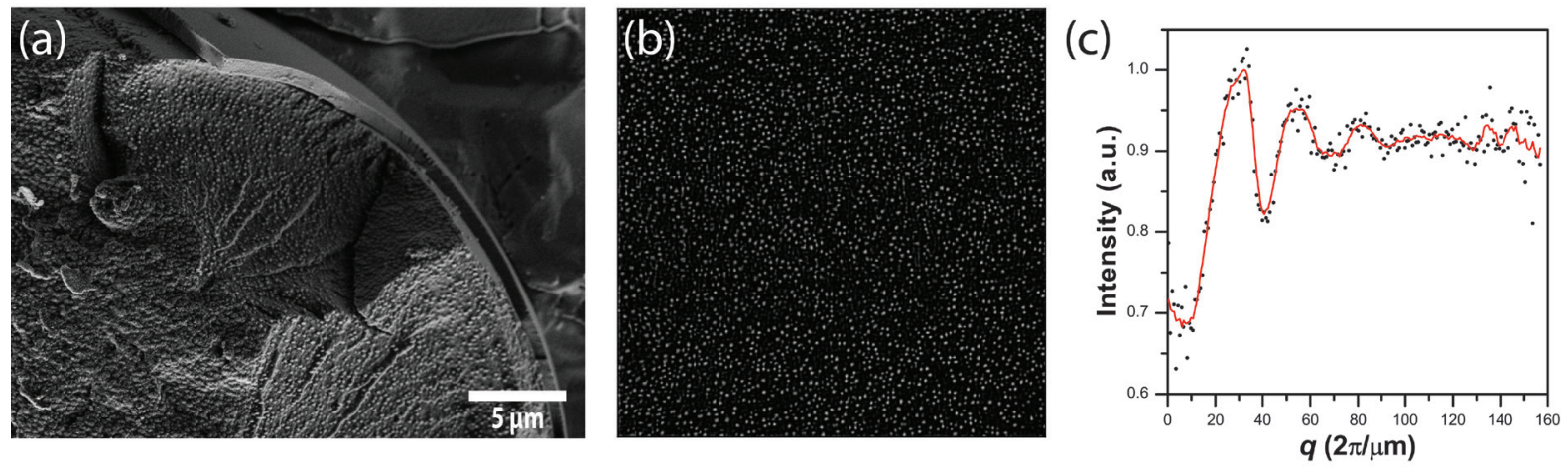

Figure S1. Investigation of the internal structure of a photonic microcapsule. (a) A SEM image of a cryogenically fractured surface of a photonic microcapsule prepared under $440 \mathrm{mOsm} / \mathrm{L}$ of osmotic pressure. (b) A binary rendering image of the SEM image in Figure 1(g) after contrast adjustment. (c) An azimuthally-averaged, 2D spatial power spectrum of the binarized image.

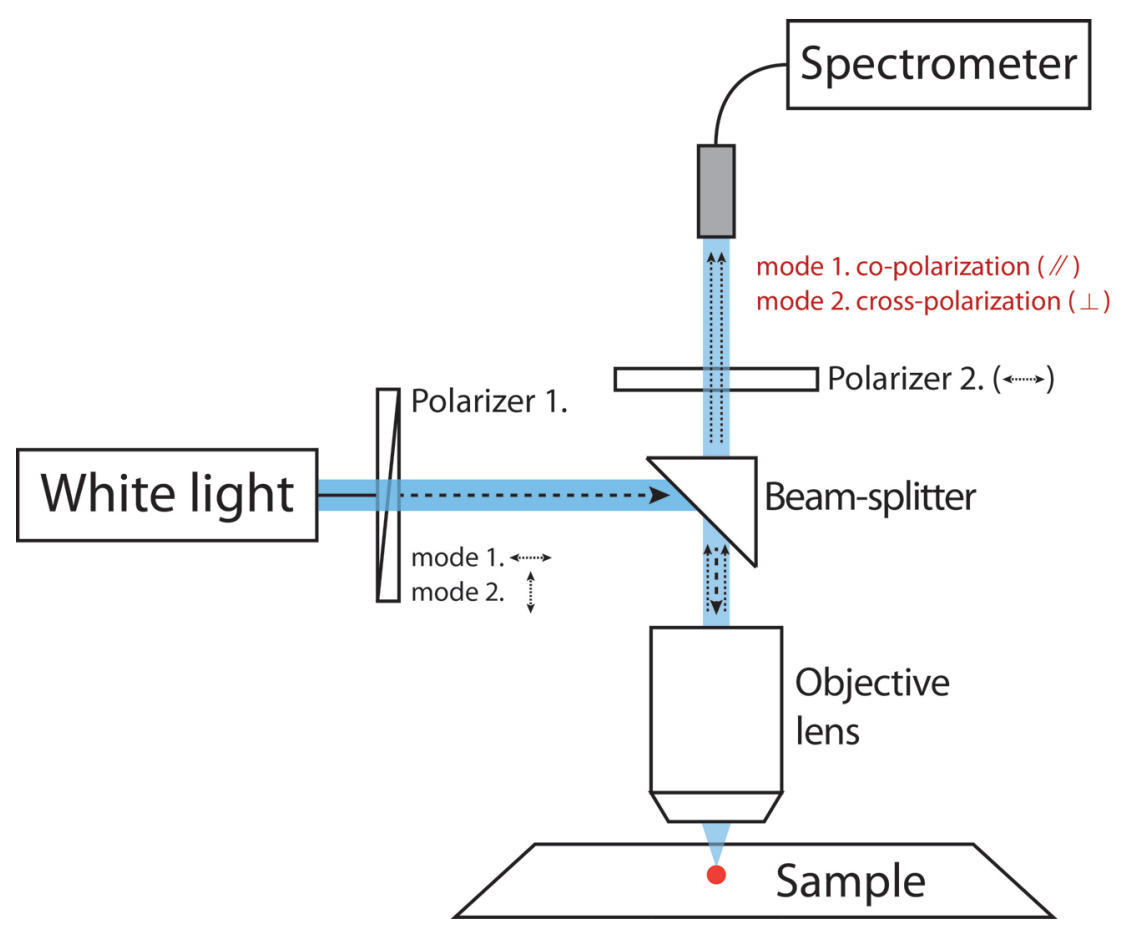

Figure S2. A schematic illustration of the instrumental setup to measure the co-polarized ( // ) and crosspolarized $(\perp)$ reflection spectra of the photonic microcapsules. 
(a)

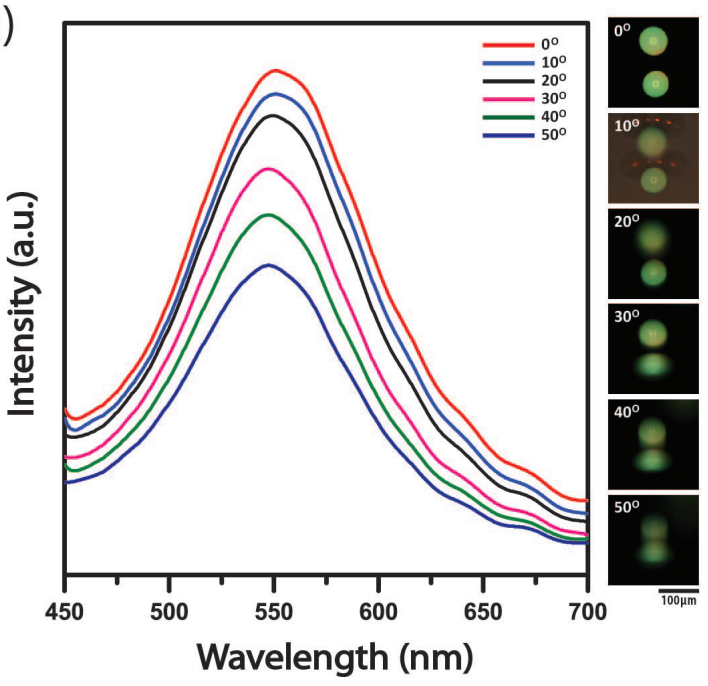

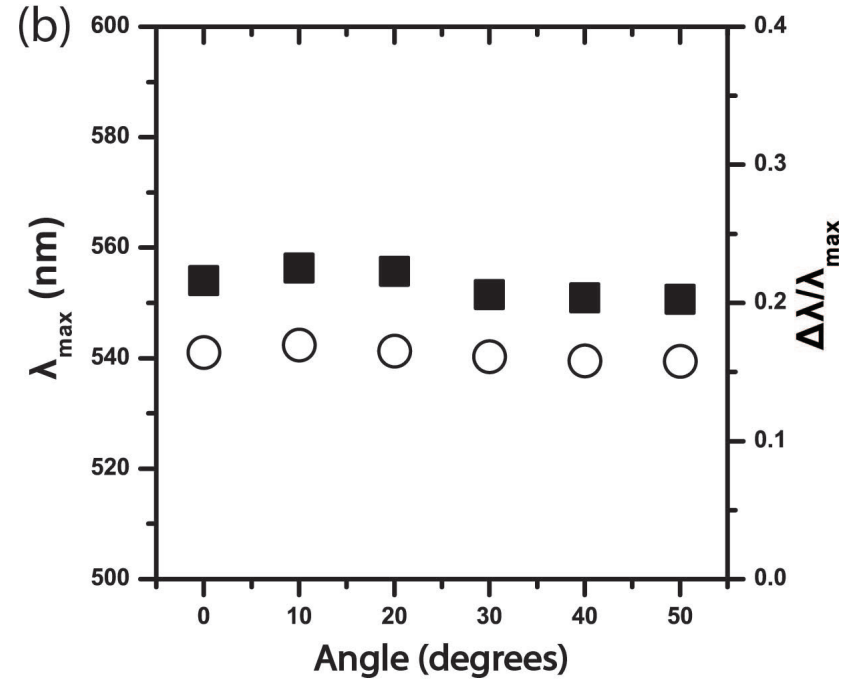

Figure S3. (a) Reflectance spectra of the photonic pigments at various viewing angles. (b) Plots of peak position ( $\lambda_{\max }$, dark squares) and normalized full-width at half maximum $\left(\Delta \lambda / \lambda_{\max }\right.$, open circles) of each peak at various angles. In this measurement, the angles of the sample stage are varied with respect to the normal to the substrate. 